\title{
Arousal modulates the motor interference effect stimulated by pictures of threatening animals
}

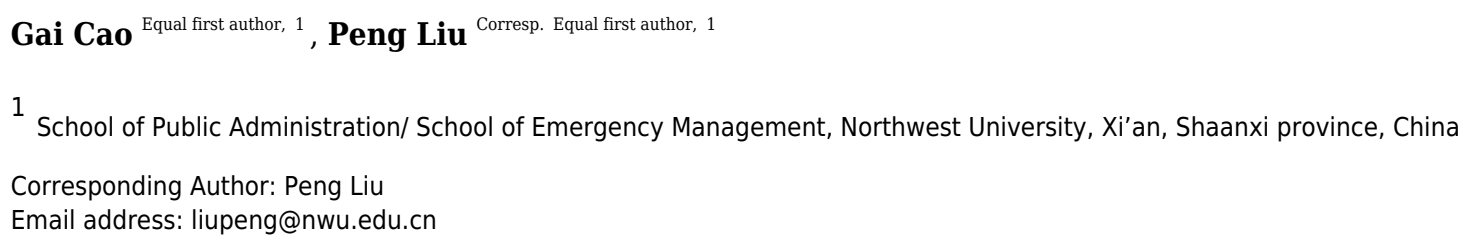

Previous research related to the motor interference effect from dangerous objects indicated that delayed responses to dangerous objects were associated with more positive parietal P3 amplitudes, suggesting that great attentional resources were allocated to evaluate the level of danger (i.e., negative valence). However, arousal covaried with valence in this research. Together with previous studies in which the P3 amplitude was found to be increased along with a higher arousal level in the parietal lobe, we raised the issue that more positive parietal P3 amplitudes might also be affected by a high arousal level. To clarify whether valence or arousal impacted the motor interference effect, this study used a motor priming paradigm mixed with a Go/NoGo task and manipulated the valence (negative, neutral and positive) and arousal (medium and high) of target stimuli. Analysis of the behavioral results identified a significant motor interference effect (longer reaction times (RTs) in the negative valence condition than in the neutral valence condition) at the medium arousal level and an increased effect size (increment of RT difference) at the high arousal level. The results indicated that negative valence stimuli may interfere with the prime elicited motor preparation more strongly at the high arousal level than at the medium arousal level. The ERP results identified larger centroparietal P3 amplitudes for the negative valence condition than for the neutral valence condition at a high arousal level. However, the inverse result, i.e., lower centroparietal P3 amplitudes for the negative valence condition than for the neutral valence condition, was observed at a medium arousal level. The ERP results further indicated that the effect size of the behavioral motor interference effect increased because subjects are more sensitive to the negative valence stimuli at the high arousal level than at the medium arousal level. Furthermore, the motor interference effect is related to the negative valence rather than emotionality of the target stimuli because different result patterns emerged between the positive and negative valence conditions. Detailed processes underlying the interaction between valence and arousal effects are discussed. 
1 Arousal modulates the motor interference effect stimulated by pictures of threatening animals

$$
\text { Gai Cao }{ }^{1} \text {, Peng Liu }{ }^{1}
$$

4 Gai Cao and Peng Liu contributed equally to this work and should be considered co-first authors.

$5{ }^{1}$ School of Public Administration/ School of Emergency Management, Northwest University,

6 Xi'an, Shaanxi province, China.

7 Corresponding Author:

8 Peng Liu

9 No. 1 Xuefu Street, Xi'an, Shaanxi province, 710127, China.

10 Email address: liupeng@nwu.edu.cn 


\section{Abstract}

Previous research related to the motor interference effect from dangerous objects indicated that delayed responses to dangerous objects were associated with more positive parietal P3 amplitudes, suggesting that great attentional resources were allocated to evaluate the level of danger (i.e., negative valence). However, arousal covaried with valence in this research. Together with previous studies in which the P3 amplitude was found to be increased along with a higher arousal level in the parietal lobe, we raised the issue that more positive parietal P3 amplitudes might also be affected by a high arousal level. To clarify whether valence or arousal impacted the motor interference effect, this study used a motor priming paradigm mixed with a Go/NoGo task and manipulated the valence (negative, neutral and positive) and arousal (medium and high) of target stimuli. Analysis of the behavioral results identified a significant motor interference effect (longer reaction times (RTs) in the negative valence condition than in the neutral valence condition) at the medium arousal level and an increased effect size (increment of RT difference) at the high arousal level. The results indicated that negative valence stimuli may interfere with the prime elicited motor preparation more strongly at the high arousal level than at the medium arousal level. The ERP results identified larger centroparietal P3 amplitudes for the negative valence condition than for the neutral valence condition at a high arousal level. However, the inverse result, i.e., lower centroparietal P3 amplitudes for the negative valence condition than for the neutral valence condition, was observed at a medium arousal level. The ERP results further indicated that the effect size of the behavioral motor interference effect increased because subjects are more sensitive to the negative valence stimuli at the high arousal level than at the medium arousal level. Furthermore, the motor interference effect is related to the negative valence rather than emotionality of the target stimuli because different result patterns emerged between the positive 
34 and negative valence conditions. Detailed processes underlying the interaction between valence

35 and arousal effects are discussed.

36 Keywords: motor interference effect, motor priming paradigm, threatening animals, arousal,

37 valence 


\section{Introduction}

Increasing production safety accidents occur in factories when workers interact with more types of machines. For example, during the process of operating a machine, dicing or saw blades in the machine may cut off a worker's finger if his or her prepared motor actions are not inhibited in time. According to a survey, human factors cause approximately $88 \%$ of work-related accidents (Huang et al., 2012). Thus, it is necessary to explore how we process our prepared motor actions to avoid touching dangerous elements in a machine, which might provide a reference for safety management that reduces the occurrence of work-related accidents from a cognitive perspective.

There has been evidence that dangerous objects may delay an individual's response speed; this phenomenon is known as the motor interference effect from dangerous objects (Anelli, Borghi, \& Nicoletti, 2012). Using a motor priming paradigm, previous research has investigated mechanisms of the motor interference effect from behavioral and event-related potential (ERP) perspectives. Anelli et al. (2012) adopted a motor priming paradigm using different means of grasping right hands as primes (a grasping or static human hand or a grasping robotic hand) and dangerous or safe objects as targets. Participants were asked to respond to artifacts or natural attributes of the targets. The results showed delayed responses for dangerous targets compared with those for safe targets, suggesting that the threat imposed by an object may conflict with an individual's prepared motor actions and thus cause slower response times (RTs). To further investigate the neuro-origin of the motor interference effect from dangerous objects, Liu et al. (2017) adopted a motor priming paradigm mixed with a Go/NoGo task. Left or right grasping hands were used as prime stimuli, and dangerous (a rectangular saw blade and a round saw blade) or safe (a ruler and a disc) objects were used as target stimuli. A green or red dot was superimposed on the center of the targets as a Go or NoGo signal, respectively. The participants were asked to 
61 prepare the ipsilateral hand responses that corresponded to the handedness of the prime. They were

62 asked to execute the prepared responses until the green dot (Go signal) emerged and to inhibit their responses while they observed the red dot (NoGo signal). The behavioral results replicated a 64 classical motor interference effect from dangerous objects, as found by Anelli et al. (2012). Moreover, the ERP results revealed a more positive parietal P3 amplitude in a dangerous condition than in a safe condition. As the parietal P3 component represents attentional resource allocation

67 (Isreal, Chesney, Wickens, \& Donchin, 1980), Liu et al. (2017) concluded that the motor interference effect originated from danger evaluation because many attentional resources were recruited to evaluate the dangerousness of the target to prevent the touching of dangerous stimuli. a dangerous target activates an aversive motivational system that may elicit an avoidance response. of stimuli (Lang, Bradley, \& Cuthbert, 1997). Valence reflects subjective appraisal of stimulus pleasantness (positive versus negative); specifically, a more negative valence stimulus may elicit a deeper activation of the aversive motivational system (Lang, Bradley, \& Cuthbert, 1990; Lang, Davis, \& Öhman, 2000). However, another important attribute (i.e., arousal, reflecting the level of activation sensitivity from calmness to excitation) of threatening stimuli was neglected by Liu et al. (2017) according to the dimensional models of emotions (Russell, 1980), resulting in the issue that arousal level covaried with valence factor. The dangerous targets (rectangular or circular sawblades) might not only activate an aversive motivational system (elicited by negative valence of the dangerous targets) but also elicit a high-level excitation state (elicited by a high arousal level of the dangerous targets) because attentional resources are automatically recruited to process the dangerous targets (Blanchette, 2006; Fox et al., 2000; Öhman, Flykt, \& Esteves, 2001; Tipples, 
84 Young, Quinlan, Broks, \& Ellis, 2002). However, the safe targets (i.e., rule or disc) might not

85 activate the aversive motivational system and only elicit a medium-level excitation state (elicited

86 by a medium arousal level of the safe targets). Furthermore, existing evidence indicates that

87 amplitudes of parietal P3 components increase along with a higher level of arousal (Cuthbert et al., 2000; Keil et al., 2002; Polich, 2007; Sabatinelli et al., 2007; Schupp et al., 2003). Therefore, the presence of more positive parietal P3 amplitudes in dangerous conditions compared with those in safe conditions, reported by Liu et al. (2017), could also be explained by the notion that the arousal level of dangerous targets was higher than that of safe targets. Additionally, an alternative explanation of the behavioral motor interference effect might still exist because positive valence stimuli were not involved in the study by Liu et al. (2017). Specifically, there has been evidence that, compared with process neutral valence stimuli, both negative and positive valence stimuli processing could increase attentional resources allocated to a task (Cuthbert et al., 2000; Keil et al., 2002). One might argue that the occurrence of the motor interference effect might be attributed to emotionality (either negative or positive valence) rather than negative valence of background stimuli because both negative and positive valence background stimuli could distract attention from the main Go/NoGo task, which might increase the RTs in dangerous conditions, as in the study by Liu et al. (2017).

To clarify whether manipulating the valence factor confounded the arousal levels in Liu et al. (2017), arousal levels should be matched when comparing results between negative and neutral valence conditions. Existing evidence indicates that arousal could modulate the valence effect on participants were instructed to search for numbers in a matrix. The number matrix was presented as superimposed on an emotional picture. They manipulated factors of valence (negative, neutral 
107 and positive levels) and arousal (medium and high levels) of the emotional pictures to investigate

108 which of the two factors had a greater impact on cognitive processing. The results indicated a

109 significant interaction between arousal and valence factors. At the medium arousal level,

110 performance was worse in the negative valence condition than in the neutral and positive valence

111 conditions. However, at the high arousal level, performance of the negative valence condition was

112 improved and became similar to the performance of the neutral and positive valence conditions.

113 The results suggested that arousal could significantly modulate the valence effect on a cognitive

114 task. Negative valence background stimuli could distract attention from the main cognitive task at

115 the medium arousal level, which impaired performance in this condition. The decrement in

116 performance could be compensated with increasing arousal levels (at the high arousal level) of the

117 background stimuli because an increased arousal level could speed up the cognitive system, which

118 in turn improved task performance in negative valence conditions to a level similar to that in

119 neutral and positive valence conditions at the high arousal level.

120 By referring to the studies from Zsido's lab, we systematically manipulated factors of

121 valence and arousal to investigate whether these two factors impact the motor interference effect

122 from dangerous objects. The study continued to use the experimental paradigm (a motor priming

123 paradigm mixed with a Go/NoGo task) adopted by Liu et al. (2017) with the following exceptions:

124 1) The background targets were selected from the International Affective Picture System (IAPS)

125 (Lang et al., 2005), in which levels of valence and arousal factors have been standardized and

126 defined. Only touchable animal pictures were selected for the background target stimuli because

127 touchable stimuli could interact with the motor preparations induced by a priming stimulus (a left

128 or right grasping hand). Moreover, selecting animal pictures as the targets could also exclude a

129 confounding factor that might be caused by different categories of targets. 2) Valence (negative, 
130 neutral and positive) and arousal (medium and high) levels of target stimuli were systematically

131 manipulated according to Zsido et al. (2020b). Positive valence was included to clarify whether

132 the motor interference effect from dangerous objects occurs because negative valence implies

133 delayed responses for background targets or whether it occurs because emotionality (either

134 negative or positive) of target stimuli distracted attention from the Go/NoGo task, which in turn

135 delays responses. Of note, the arousal levels were matched among negative, neutral and positive

136 valence in the animal category of target stimuli, and the mean scores of the high arousal level

137 (mean $=5.68)$ are lower than those of in the study by Zsido et al. $(2020 \mathrm{~b})($ mean $=7.57)$. The

138 effectiveness of the manipulation is discussed in the General Discussion. 3) Emotional pictures

139

140

141

142

143

144

145

146

147

148

149

150

151

152

were used as backgrounds with a Go/NoGo signal (yellow capital letters "M" or "W") superimposed on the center. Participants were instructed to prepare the ipsilateral hand responses that corresponded to the handedness of the prime and to decide whether to execute the prepared responses according to the Go/NoGo signal. Responding to the superimposed targets imitated a situation in which executing a prepared response encountered an emergent dangerous stimulus because emotion is triggered by accidental stimuli in usual life settings (Delplanque, Silvert, Hot, \& Sequeira, 2005; Yuan et al., 2007). The current design that did not require subjects to evaluate emotion overtly (the emotional stimuli are used as backgrounds) may have allowed emotional responses in the laboratory setting to more closely imitate life experiences.

The hypotheses were based on the work of Zsido et al. (2020b), namely, that arousal could modulate the strength of the motor interference effect. Specifically, at the medium arousal level, the mean RTs in the negative valence condition should be longer than those in the neutral and positive valence conditions because negative valence background stimuli could distract attention from the Go/NoGo signals at the medium arousal level. In contrast, high arousal stimuli might 
153 divert attentional resources to the background targets, which may enhance perceptual

154 representation of negative valence targets. Participants might suppress their prepared responses

155 more strongly because many threatening details could be analyzed by the increased attentional

156 resources in the high arousal and negative valence condition. Therefore, a larger effect size (mean

157 RT in the negative valence condition minus those in the neutral and positive valence conditions)

158 should emerge at the high arousal level compared with the medium arousal level. For ERPs,

159 according to Liu et al. (2017), who suggested that the valence factor could significantly contribute

160 to the P3 amplitudes, more positive P3 amplitudes should emerge in the negative valence condition

161 than in the neutral and positive valence conditions at the medium arousal level. Moreover, the

162 differences in the P3 amplitudes between the negative and neutral valence conditions and those

163 between the negative and positive valence conditions should be larger at the high arousal level

164 than the medium arousal level.

1652 Method

$166 \quad 2.1$ Participants

167 To obtain robust results, 76 right-handed subjects were recruited to participate in the

168 experiment. Note that the number of subjects was more than that of a prior simple size estimation

169 (7 subjects) with a large effect size $(f=0.4)$ and 0.95 statistical power. Subjects No. 6 and No. 11

170 were excluded from the data analysis because of a reference channel error. The remaining 74

171 subjects (thirty males), ranging in age from 18 to 26 years (mean age $=21.91$ ), were included in

172 the data analysis. All participants had normal or corrected-to-normal visual acuity. They also

173 reported an absence of neurological disorders. They provided written informed consent before

174 participating the experiment and were compensated with RMB 50 while finishing the experiment.

175 The experiment was approved by the Medical Ethics Committee at Northwest University. 


\subsection{Materials and apparatus}

Left or right hand with a partial forearm pictures (subtending a visual angle $13^{\circ}$ horizontally and $11^{\circ}$ vertically) were used as primes, which aimed to activate a corresponding directional response readiness (Liu, Cao, Chen, \& Wang, 2017). To imitate a spatially matched grasping situation, primes were presented $2^{\circ}$ to the left or right of the fixation point. Centrally presented targets were combined pictures with a Go/NoGo signal (yellow capital letters "M" or "W" subtending a visual angle $2^{\circ}$ horizontally and $2^{\circ}$ vertically) superimposed on the center of an emotional picture (the size of raw pictures was adjusted to a visual angle $12^{\circ}$ horizontally and $9^{\circ}$ vertically). Emotional pictures were selected from the IAPS (Lang et al., 2005) with the following criteria: 1) touchable stimuli were selected as targets to interact with the motor preparations induced by priming stimuli and 2) all of the selected stimuli belonged to one category (i.e., animal) to exclude stimulus categories as a confounding factor. Accordingly, we selected 18 animal pictures (Table 1) as backgrounds with systematically manipulated valence and arousal levels (Table 2). Two two-way analyses of variances (ANOVAs), as a function of valence and arousal, were separately performed for the valence and arousal scores. Analysis of the valence scores revealed a significant main effect of valence $\left[F(2,12)=65.94, p<0.001, \eta^{2}{ }_{p}=0.92\right]$, together with an insignificant main effect of arousal $\left[F(1,12)=0.001, p=0.99, \eta_{p}^{2}=0.001\right]$ and insignificant interaction $\left[F(2,12)=0.04, p=0.97, \eta_{p}^{2}=0.006\right]$. Moreover, analysis of the arousal scores revealed a significant main effect of arousal $\left[F(1,12)=42.13, p<0.001, \eta_{p}^{2}=0.78\right]$, together with an insignificant main effect of valence $\left[F(2,12)=0.67, p=0.53, \eta^{2}{ }_{p}=0.10\right]$ and insignificant interaction $\left[F(2,12)=0.08, p=0.92, \eta_{p}^{2}=0.01\right]$. The results suggested that both valence and arousal levels were systematically manipulated, with equivalent valence ratings between medium and high arousal levels and equivalent arousal ratings among negative, positive 
199

200

201

202

203

204

205

206

207

208

209

210

211

212

213

214

215

216

217

218

219

220

221

and neutral valence levels.

[Please insert Tables 1 and 2 about here]

Data were recorded as previously described in Liu et al. (2017). Specifically, stimulus presentation was driven by an E-Prime software (version 2.0, Psychology Software Tools, Inc. Pittsburgh, PA, USA) on a standard PC linked to a 17- inch CRT monitor (60-Hz refresh rate). Electroencephalogram (EEG) data were recorded by a NeuroScan system (NeuroScan, Inc.). A Neuroscan Synamp 2 amplifier with a $64 \mathrm{Ag} / \mathrm{AgCl}$ electrode cap mounted according to an extended international 10-20 system was used to continuously record EEG data.

\subsection{Procedure}

To reduce eye fatigue, all stimuli were presented on a black background. Each trial was initiated from a 300-ms fixation cross to alert participants to concentrate on the screen. Then, a 300-ms blank screen, a 200-ms left- or right-hand prime, another 50-ms blank screen, and a 1000ms target were successively presented. Note that the target display was terminated if the response was executed within 1,000 ms. The intertrial interval was randomized within 1,400-1,600 ms.

The participants were seated in front of a laboratory table in a dimly lit chamber. A bracket fixed on the table held their chin to maintain central eye fixation and to fix a computer screen placed $60 \mathrm{~cm}$ in front of their eyes throughout the experiment. They were instructed to respond according to the Go/NoGo signal ("M" or "W"), which was superimposed on the emotional pictures. Specifically, they were instructed to prepare a left- or right-hand response that corresponded to the handedness of the prime and not to execute the response until a Go signal appeared. Half of the participants were asked to execute the prepared response as fast and accurately as possible whenever the letter "M" (Go) emerged and to withhold the prepared response when presented with the letter "W" (Nogo). The response rule was counterbalanced in 
222 the other half of the participants, with the letter "W" as a Go signal and the letter "M" as a NoGo

223 signal. The participants were instructed to execute a left-hand response by pressing the "F" key

224 using the index finger of their left hand and to execute a right-hand response by pressing the "J"

225 key using the index finger of their right hand on an English keyboard. In contrast, when a NoGo

226 signal appeared, the participants were instructed to withhold the prepared response.

227 The design manipulated valence (negative, neutral and positive), arousal (medium versus

228 high) and Go/NoGo factor (Go versus NoGo). The formal task contained 1152 trials, which

229 included 3 levels of valence $\times 2$ levels of arousal $\times 2$ levels of Go/NoGo $\times 96$ repetitions. In each

230 condition, left or right handedness of the prime was assigned in equal proportions. At the

231 beginning, a 12-trial practice was performed. The formal task would begin unless the participant

232 correctly answered over 11-trials in the practice phase. Each block contained 128 trials. The

233 participants could take a rest between the blocks until they were ready for the next block.

234

235

236

237

238

239

240

241

242

243

244

\subsection{EEG recording and processing}

The EEG data were recorded with the signals bandpass-filtered at $0.05-100 \mathrm{~Hz}$ and referenced to the tip of the nose. To ensure signal quality, the impedance of the electrodes was maintained at less than $5 \mathrm{k} \Omega$ throughout the experiment. The sampling rate was $500 \mathrm{~Hz}$. The recorded EEG data were preprocessed using the EEGLAB toolbox (Delorme \& Makeig, 2004) according to the following steps: (1) The continuous EEG data were resampled at $250 \mathrm{~Hz}$. (2) The resampled EEG data were high-pass filtered at $0.1 \mathrm{~Hz}$ and low-pass filtered at $30 \mathrm{~Hz}$. (3) The EEG data were segmented and time-locked to the target onset. The duration of each epoch was 3,000 ms with a baseline of 1,000 ms before the target onset. (4) The epoched data were corrected using the mean amplitude of the baseline. (5) The behavioral data were merged into the epoched data, and the incorrect trials were deleted. (6) Bad channels were deleted. (7) The epoched data 
245 contaminated by eye blinks and eye movements were corrected using the independent component

246 analysis (ICA) algorithm (Delorme \& Makeig, 2004). (8) The deleted channels were interpolated

247 using the EEGLAB toolbox. (9) The epochs were re-referenced to the mean of the bilateral mastoid

248 electrodes, and (10) the epochs with large artifacts were detected by eye and manually deleted.

249 Automatic artifact detection was then performed with deletion of the trials containing amplitudes

250 less than $-100 \mu \mathrm{V}$ or more than $100 \mu \mathrm{V}$. Consequently, $2.5 \%$ of the epochs were rejected as

251 contaminated during preprocessing across all subjects and conditions. The mean number of

252 artifact-free trials obtained for each condition stabilized between 93 and 94, which ensured a valid

253 trial amount for each condition.

$\mathrm{ms}$ before and $900 \mathrm{~ms}$ after the target onset. A flat time window (-300 to $-200 \mathrm{~ms}$ before the target

256

257

258

259

260

261

262

263

264

265

266

267

onset) was selected as a baseline to correct the new epochs. Then, the extracted average waveforms

for each participant and condition were used to calculate the grand-average waveforms.

\subsection{Statistical analysis}

\subsubsection{Behavioral data}

Mean RTs and mean error rates for each condition were averaged separately for each participant. Note that only the RTs for correct responses in Go trials were involved in the RT analysis. Before the analysis, Kolmogorov-Smirnov tests of normality were performed on RTs and error rates for each condition. The results indicated that mean RTs were normally distributed for all conditions. Accordingly, the mean RTs were analyzed by a two-way repeated-measures ANOVA as a function of valence (negative, neutral and positive) and arousal (medium versus high). However, the Kolmogorov-Smirnov tests indicated that the mean error rates deviated from normality for all conditions $(p \mathrm{~s}<0.01)$. Accordingly, logarithmic mean correct rates (because 0 
268

269

270

271

272

273

274

275

276

277

278

279

280

281

282

283

284

285

286

287

288

289

290

cannot be log-transformed, we used correct rates instead of error rates) were analyzed by a threeway repeated-measures ANOVA as a function of valence (negative, neutral and positive), arousal (medium versus high) and Go/NoGo factor (Go versus NoGo).

\subsubsection{ERP data}

To utilize more channel signals, the centroparietal scalp regions of interest (SROIs, the average of the $\mathrm{C} 5, \mathrm{C} 3, \mathrm{C} 1, \mathrm{Cz}, \mathrm{C} 2, \mathrm{C} 4, \mathrm{C} 6, \mathrm{CP} 5, \mathrm{CP} 3, \mathrm{CP} 1, \mathrm{CPz}, \mathrm{CP} 2, \mathrm{CP} 4, \mathrm{CP} 6, \mathrm{P} 5, \mathrm{P} 3, \mathrm{P} 1, \mathrm{Pz}$, P2, P4 and P6 electrodes) were defined according to the topographic maps (more details are presented in Figure 1), where the electrodes were maximally activated. The dependent variables comprised centroparietal P3 amplitudes, which were calculated based on the mean amplitude between 300 and $500 \mathrm{~ms}$ for Go and that between 400 and $600 \mathrm{~ms}$ for NoGo trials. Note that only P3 amplitudes were used as an ERP index because Liu et al. (2017) identified that the neural processing of the motor interference effect from dangerous objects is reflected by the amplitudes of the late component (i.e., P3 amplitudes) rather than by those of the early (P1 and N1) and middle (P2 and N2) latency components. The independent variables were valence (negative, neutral and positive), arousal (medium versus high), and Go/NoGo factor (Go versus NoGo). KolmogorovSmirnov tests of normality were also performed on P3 amplitudes in each condition. The results indicated that distributions of $\mathrm{P} 3$ amplitudes did not deviate from normality. Accordingly, a threeway repeated-measures ANOVA was used to analyze the effects of the independent variables. The degrees of freedom of the $F$-ratio were corrected using the Greenhouse-Geisser method, and multiple comparisons were adjusted by the Bonferroni method in the analyses. The effect sizes are presented as partial eta-squared values $\left(\eta^{2}\right)$ for the ANOVA and as Cohen's $d$ s for the $t$-tests.

[Please insert Figure 1 about here]

\section{Results}


291

292

293

294

295

296

297

298

299

300

301

302

303

304

305

306

307

308

309

310

311

312

313

\subsection{Behavioral results}

The analysis of RTs revealed significant main effects of valence $[F(2,146)=50.50, p<$ $\left.0.001, \eta_{p}^{2}=0.41\right]$ and arousal $\left[F(1,73)=22.01, p<0.001, \eta_{p}^{2}=0.23\right]$, together with a significant two-way interaction between valence and arousal $\left[F(2,146)=39.05, p<0.001, \eta^{2}{ }_{p}=0.35\right]$ (Figure 2). Subsequent one-way ANOVAs indicated significant main effects of valence at both high $[F(2$, $\left.146)=77.59, p<0.001, \eta_{p}^{2}=0.52\right]$ and medium $\left[F(2,146)=16.91, p<0.001, \eta_{p}^{2}=0.19\right]$ arousal levels. Post hoc tests with Bonferroni correction indicated that at the high arousal level, RTs for the negative (492 $\pm 54 \mathrm{~ms} ; p<0.001$, Cohen's $d=1.19)$ and positive valence $(492 \pm 51 \mathrm{~ms} ; p<$ 0.001, Cohen's $d=1.31)$ conditions were slower than those for the neutral valence condition (474 $\pm 53 \mathrm{~ms}$ ), and the difference between the negative and positive valence conditions did not reach significance $(p=1.00$, Cohen's $d=0.01)$. In contrast, at the medium arousal level, responses for the negative valence condition $(496 \pm 52 \mathrm{~ms})$ were slower than those for the positive (486 \pm 53 ms; $p<0.001$, Cohen's $d=0.64)$ and neutral valence $(488 \pm 50 \mathrm{~ms} ; p<0.001$, Cohen 's $d=0.47)$ conditions, and the difference between the positive and neutral valence conditions did not reach significance $(p=0.76$, Cohen's $d=0.13)$.

[Please insert Figure 2 about here]

Regarding error rates (Figure 3), the analysis of log-transformed correct rates revealed significant main effects of valence $\left[F(2,146)=4.93, p=0.01, \eta_{p}^{2}=0.06\right]$ and arousal $[F(1,73)$ $\left.=5.04, p=0.03, \eta_{p}^{2}=0.07\right]$. Furthermore, a significant two-way interaction between valence and arousal $\left[F(2,146)=3.65, p=0.03, \eta^{2}{ }_{p}=0.05\right]$ together with a significant three-way interaction among valence, arousal and Go/NoGo factor $\left[F(2,146)=5.03, p=0.009, \eta_{p}^{2}=0.06\right]$ was identified. Analysis of the simple effect of the significant three-way interaction revealed a significant two-way interaction between valence and arousal in Go trials $[F(2,146)=7.00, p=$ 
$\left.3140.02, \eta^{2}{ }_{p}=0.09\right]$. Subsequent one-way ANOVAs indicated a nonsignificant main effect of valence

315 at the high arousal level $\left[F(2,146)=0.39, p=0.65, \eta_{p}^{2}=0.005\right]$. However, the main effect of

316 valence reached significance at the medium arousal level $\left[F(2,146)=7.54, p=0.001, \eta_{p}^{2}=0.09\right]$.

317 The post hoc analysis indicated that response errors for the negative valence condition $(1.72 \pm 2.40$

$318 \%)$ were more than those for the positive $(1.04 \pm 1.49 \% ; p=0.003$, Cohen's $d=0.41)$ and neutral

319 valence $(1.11 \pm 1.85 \% ; p=0.02$, Cohen's $d=0.34)$ conditions, and the difference between the

320 positive and neutral valence conditions did not reach significance $(p=1.00$, Cohen's $d=0.05)$. In

321 contrast, analysis of the simple effect indicated significant main effects of valence $[F(2,146)=$

$\left.3224.57, p=0.01, \eta_{p}^{2}=0.06\right]$ and arousal $\left[F(1,73)=7.40, p=0.008, \eta_{p}^{2}=0.09\right]$ in NoGo trials. The

323 post hoc analysis of valence indicated that response errors for the positive $(1.19 \pm 1.24 \% ; p=$

3240.04 , Cohen's $d=0.30)$ and negative $(1.20 \pm 1.29 \% ; p=0.057$, Cohen's $d=0.28)$ valence

325 conditions were more and nearly more than those for the neutral valence condition $(0.82 \pm 1.09$

$326 \%$, respectively. The post hoc analysis of arousal indicated that response errors for the high arousal

327 condition $(0.91 \pm 1.03 \%)$ were less than those for the medium arousal condition $(1.23 \pm 1.17 \%)$.

[Please insert Figure 3 about here]

329

330

331

332

333

334

335

336

\subsection{ERP results}

Grand averages of target-locked ERPs are presented in Figure 4. The three-way repeatedmeasures ANOVA results of the centroparietal P3 amplitudes revealed insignificant main effects of valence $\left[F(2,146)=2.32, p=0.10, \eta_{p}^{2}=0.03\right]$, arousal $\left[F(1,73)=1.75, p=0.19, \eta^{2}{ }_{p}=0.02\right]$ and Go/NoGo factors $\left[F(1,73)=3.21, p=0.08, \eta_{p}^{2}=0.04\right]$. The two-way interaction between valence and arousal reached significance $\left[F(2,146)=18.55, p<0.001, \eta^{2} p=0.20\right]$. However, the two-way interaction between valence and Go/NoGo $\left[F(2,146)=2.34, p=0.10, \eta_{p}^{2}=0.03\right]$ and that between arousal and Go/NoGo $\left[F(1,73)=0.75, p=0.39, \eta_{p}^{2}=0.01\right]$, together with the three- 
337 way interaction among all factors $\left[F(2,146)=2.84, p=0.06, \eta_{p}^{2}=0.04\right]$, did not reach

338 significance. To assess the valence effect on different arousal levels, we performed two one-way ANOVAs as a function of valence at the high and medium arousal levels. Specifically, at the 340 medium arousal level, the main effect of valence reached significance $[F(2,146)=4.41, p=0.01$, $\left.341 \eta_{p}^{2}=0.06\right]$. Post hoc tests with Bonferroni correction indicated that the P3 amplitudes for the 342 positive valence $(2.81 \pm 2.71 \mu \mathrm{V} ; p=0.04$, Cohen's $d=0.30)$ and neutral valence $(2.81 \pm 3.03$ $343 \mu \mathrm{V} ; p=0.03$, Cohen's $d=0.31$ ) conditions were more positive than those for the negative valence condition $(2.53 \pm 2.87 \mu \mathrm{V})$, and the difference between the positive and neutral valence conditions did not reach significance $(p=1.00$, Cohen's $d=0.003)$. At the high arousal level, the main effect of valence reached significance $\left[F(2,146)=18.43, p<0.001, \eta^{2}{ }_{p}=0.20\right]$. Post hoc tests with Bonferroni correction indicated that the P3 amplitude for the negative valence condition $(3.11 \pm$ $2.87 \mu \mathrm{V})$ was more positive than that for the neutral valence $(2.77 \pm 2.72 \mu \mathrm{V} ; p=0.004$, Cohen's $d=0.38)$ and positive valence $(2.52 \pm 2.77 \mu \mathrm{V} ; p<0.001$, Cohen's $d=0.70)$ conditions. Moreover, the P3 amplitude for the neutral valence condition was more positive than that for the positive valence condition ( $p=0.03$, Cohen's $d=0.32)$.

[Please insert Figure 4 about here]

353

354

355

356 357 358 359

\section{Discussion}

\subsection{Overview of the study}

This study investigated whether different arousal levels (medium versus high) could modulate the motor interference effect from dangerous objects. The design adopted a motor priming paradigm mixed with a Go/NoGo task, which was consistent with Liu et al. (2017), and further manipulated the valence and arousal of target stimuli referred to Zsido et al. (2020b). The target stimuli were selected from the IAPS with a yellow capital letter "M" or "W" superimposed 
360 on them as a Go or NoGo signal. Participants were instructed to prepare the ipsilateral hand 361 responses that corresponded to the handedness of the prime and to decide to execute the prepared

362

363

364

365

366

367

368

369

370

371

372

373

374

375

376

377

378

379

380

381

382 responses or not according to the Go/NoGo signals.

The hypotheses proposed a modulation effect of different arousal levels on valence factor. Specifically, the motor interference effect should emerge at the medium arousal level with the responses in the negative valence condition executing slower than those in the neutral and positive valence conditions. In contrast, at the high arousal level, the strength of the motor interference effect should increase compared with the medium arousal level. The ERP results predicted more positive P3 amplitudes in the negative valence condition than in the neutral and positive valence conditions at the medium arousal level. Moreover, the differences in the P3 amplitudes between the negative and neutral valence conditions and those between the negative and positive valence conditions should be larger at the high arousal level than the medium arousal level.

The behavioral results of RTs revealed a significant motor interference effect with longer RTs for the negative valence condition than for the neutral and positive valence conditions at the medium arousal level. The results supported the hypothesis that the motor interference effect originated from negative valence rather than the emotional attribute (either negative or positive valence) of the target stimuli. Specifically, only negative background stimuli could distract attention from the main Go/NoGo task at the medium arousal level. At the high arousal level, a significant motor interference effect was also observed with longer RTs for the negative valence condition than for the neutral valence condition. Moreover, the effect size (mean RTs of the negative valence condition minus that of the neutral valence condition) was examined by the twoway interaction between valence (negative versus neutral) and arousal (medium versus high) in Go trials. The results revealed a significant two-way interaction $\left[F(1,73)=20.50, p<0.001, \eta^{2}{ }_{p}\right.$ 
$383=0.22]$, which supports the hypothesis that the effect size of the motor interference effect at the

384 high arousal level $(19 \mathrm{~ms})$ is significantly larger than that at the medium arousal level $(8 \mathrm{~ms})$. The

385 results support the hypothesis that high arousal stimuli distract many attentional resources from

386 processing the background targets, which may enhance threatening details implied in the negative

387 valence targets. Responses are interfered more strongly in the negative valence condition at the

388 high arousal level than the medium arousal level. However, the difference in RTs between the

389 negative and positive valence conditions did not reach significance, which violates the hypothesis

390 and might suggest that both the negative and positive background stimuli distract attention from

391 the Go/NoGo signals at the high arousal level. The violation of the hypothesis was discussed

392 together with the ERP results in the following paragraphs.

393 The ERP results present a deeper investigation of the motor interference effect. At the high

394 arousal level, more positive P3 amplitudes emerged in the negative valence condition than the

neutral valence condition. As the centroparietal P3 amplitude reflects attentional resource

allocation (Isreal et al., 1980), the results support the hypothesis that many attentional resources

are assigned to the negative valence condition. Because responding to the Go/NoGo signal is a simple task, remnant attentional resources could be allocated to the background stimuli. This would enhance the processing of the threatening details implied in the negative valence targets, enabling the more dangerous targets. As a more negative valence stimulus may elicit a deeper activation of the aversive motivational system (Lang et al., 1990, 2000), the strength of the motor interference effect could be increased. However, at the medium arousal level, less positive P3 amplitudes emerged in the negative valence condition than the neutral valence condition. Assuming that consistent attentional resources are consumed by the Go/NoGo signals, fewer 405 attentional resources could be allocated to the negative valence background stimuli at the medium 
406

407

408

409

410

411

412

413

arousal level than those at the high arousal level, enabling the negative valence targets to be less dangerous; thus, the strength of the motor interference effect could be decreased.

The positive valence was included in the experimental design to exclude an alternative explanation of whether the motor interference effect originates from the emotionality of the background stimuli. Similar result patterns were expected between the positive and negative valence conditions according to this hypothesis. Although identical RTs were observed between the negative and positive valence conditions at the high arousal level, the P3 amplitudes significantly differed. The ERP results indicated that fewer attentional resources were assigned to the positive valence stimuli than to the negative valence stimuli at the high arousal level. A possible reason was proposed based on the valence-arousal conflict theory (Feng et al., 2012; Robinson et al., 2004). The theory suggested that negative valence stimuli evoke the aversive motivational system at a high arousal level. Additionally, subjects might have been more sensitive to the negative valence than the positive valence stimuli, which decreased the P3 amplitude of the positive valence condition. Moreover, the positive valence stimuli force subjects to approach the stimuli (Feng et al., 2012; Robinson et al., 2004). Thus, a conflict exists between high arousal elicited aversive motivation and positive valence evoked approach motivation, which might delay the responses to the positive valence stimuli at the high arousal level. The explanation was also supported by the results at the medium arousal level. According to the valence-arousal conflict theory, the appetitive motivational system will gradually replace the aversive motivational system to dominate emotional cognitive processing along with lower arousal levels. Subjects might be more sensitive to the positive valence than the negative valence stimuli, which increased the P3 amplitude of the positive valence condition at the medium arousal level. When the motivational tendency is congruent between appetitive motivation elicited by medium arousal and approach 
429

430

431

432

433

434

435

436

437

438

439

440

441

442

443

444

445

446

447

motivation evoked by positive valence, responses to the positive valence stimuli are accelerated and become close to the neutral valence stimuli at a medium arousal level because the conflict diminishes in the positive valence and medium arousal condition.

The valence-arousal conflict theory could also provide an alternative explanation to the delayed responses and the less positive P3 amplitudes in the negative valence condition compared with those in the neutral valence condition at the medium arousal level. As the appetitive motivational system gradually replaces the aversive motivational system along with lower arousal levels, subjects might be less sensitive to the negative valence stimuli than the neutral and positive valence stimuli, which decreased the P3 amplitude of the negative valence condition at the medium arousal level. Moreover, the motivational tendency is incongruent between appetitive motivation elicited by medium arousal and aversive motivation evoked by negative valence, which might delay the responses to the negative valence stimuli at the medium arousal level. The validation of this alternative explanation requires further investigation.

Note that error rates are not discussed because 1) they are insensitive to the variations of valence and arousal levels. Few errors are made in the simple Go/NoGo task (the mean error rates for each condition were less than $1.72 \%$ ), which may have caused a ceiling effect; and 2) analyses of the error rates showed only small to medium effect sizes, which might signal false-positive results.

\subsection{Relationship between the current study and the study by Liu et al. (2017)}

The current study was conducted to clarify whether different arousal levels (medium versus high) could modulate the motor interference effect. We hypothesized that arousal levels could modulate valence effect on the behavioral and ERP results. The results revealed a larger motor interference effect at the high arousal level than that at the medium arousal level. Moreover, the 
452 centroparietal P3 amplitudes were more positive in the negative valence condition than the neutral

453 valence condition at the high arousal level. Nevertheless, the results of the centroparietal P3

454 amplitudes were reversed at the medium arousal level. The results expand on those obtained by

455 Liu et al. (2017) by demonstrating that the more positive parietal P3 amplitudes in the dangerous

456 condition than those in the safe condition likely emerged because the dangerous targets not only

457 activated an aversive motivational system (elicited by a negative valence level) but also enhanced

458 the strength of the activation by their high arousal attributes. Only in this manner could the parietal

459 P3 amplitudes be more positive in the dangerous condition than the safe condition in the study by

460 Liu et al. (2017).

Analysis of the centroparietal P3 amplitudes did not reveal significant interactions with the

462

463

464

465

466

467

468

469

470

471

472

473

474
Go/NoGo factor. The results suggest that valence and arousal information is processed regardless of whether the prepared responses were executed (Go trials) or not (NoGo trials). The results are inconsistent with those of Liu et al. (2017), who found a significant dangerous effect (i.e., a more positive parietal P3 amplitude in dangerous conditions than in safe conditions) only when the prepared responses were executed (Go trials). In the NoGo trials, the dangerous effect diminished. The reasons for the different results between the two studies might be due to different perceptual attributes of the background stimuli. Specifically, the colorful animal pictures adopted in the current study are perceptually richer than the gray modern pictures adopted by Liu et al. (2017). The former pictures might reflect possible experiences in daily life, which enables subjects to be more sensitive to the valence and arousal information of the background picture in both the Go and NoGo trials.

4.3 Difference between the study by Zsido et al. (2020b) and the current study

The current study manipulated valence and arousal factors in accordance with the study by

Peer) reviewing PDF | (2020:08:52283:1:1:NEW 15 Dec 2020) 
475 Zsido et al. (2020b), identical result patterns were observed at the medium arousal level that

476 responses were delayed when facing a negative valence compared with a neutral valence

477 background stimulus. However, the results differed at the high arousal level. The enlarged RT 478 differnece between the negative and neutral valence conditions at the high arousal level, observed 479 in the current study, however diminished in Zisdo et al. (2020b). The reason for the discrepancy 480 might attribute to the different difficulties of the main task. Specifically, searching for numbers in 481 a matrix required more attentional resources compared with the Go/NoGo task. Increased 482 attentional resources elicited by the high arousal background stimuli could be assigned to the 483 searching numbers task in priority, which improved performance on the main task. In contrast, 484 discriminating the Go/NoGo signals only required a few attentional resources, and the remaining 485 resources could be assigned to the background stimuli, which enhanced the processing of 486 threatening details implied in the negative valence stimuli. Thus, the motor interference effect was 487 enhanced at the high arousal level in the current study.

488 4.4 Limitations

Although the difference in the arousal scores between the medium $($ mean $=4.67)$ and high arousal $($ mean $=5.68)$ levels reached significance, the difference was relatively small compared with that in Zsido et al. (2020b) because the arousal levels were matched among negative, neutral and positive valences in the animal category of the background stimuli. The results revealed a significant modulation effect of the arousal factor, which verified the effectiveness of the manipulation in distinguishing the medium and high arousal levels. However, we believe that the arousal effect could be more salient if the design increased the difference between the medium and high arousal level. This is a limitation of the current study and should be avoided in further 497 research. 
498

499

500

501

502

503

504

505

506

507

508

509

510

511

512

513

514

515

516

517

518

519

520

\section{Conclusion}

In summary, the current study investigated whether the arousal of target stimuli could modulate the motor interference effect. The behavioral results revealed a significant motor interference effect (mean RTs in the negative valence condition minus those in the neutral valence condition) at the medium arousal level, and the effect size increased at the high arousal level. The results support the hypothesis that the motor interference effect could be modulated by different arousal levels of the stimuli with an increment of the motor interference effect along with a higher arousal level. The results indicate that negative valence stimuli may interfere with the prime elicited motor preparation more strongly at the high arousal level than at the medium arousal level. The underlying cognitive processes are reflected by the results of the centroparietal P3 amplitudes, which indicate that the effect size of the motor interference effect increased because subjects are more sensitive to the negative valence stimuli at the high arousal level than at the medium arousal level, and the activation of the aversive motivational system increased with increasing arousal level. Furthermore, the motor interference effect is related to the negative valence rather than emotionality of the target stimuli because different result patterns emerged between the positive and negative valence conditions. We attempt to relate the findings of the current study to safety management; specifically, increasing the arousal of dangerous stimuli could activate the aversive motivational system. Workers may be more sensitive to dangerous elements in machines when these elements are presented at a high arousal level (e.g., painting bold colors on dangerous elements). Thus, dangerous elements inducing a high state of arousal may increase the motor interference effect, which in turn reduces work-related accidents because responses to dangerous elements have been inhibited.

\section{Acknowledgments}


Provincial Education Department under grant number 19JK0827.

523

524

525

526

527

\section{References}

Anelli, F., Borghi, A. M., \& Nicoletti, R. (2012). Grasping the pain: Motor resonance with dangerous affordances. Consciousness and Cognition, 21(4), 1627-1639. https://doi.org/10.1016/j.concog.2012.09.001 (Original work published)

Blanchette, I. (2006). Snakes, spiders, guns, and syringes: How specific are evolutionary constraints on the detection of threatening stimuli? Quarterly Journal of Experimental Psychology, 59(8), 1484-1504. https://doi.org/10.1080/02724980543000204 (Original work published)

Cuthbert, B. N., Schupp, H. T., Bradley, M. M., Birbaumer, N., \& Lang, P. J. (2000). Brain potentials in affective picture processing: Covariation with autonomic arousal and affective report. Biological Psychology. https://doi.org/10.1016/S0301-0511(99)00044-7 (Original work published)

Delorme, A., \& Makeig, S. (2004). EEGLAB: an open source toolbox for analysis of single-trail EEG dynamics including independent component analysis. Journal of Neuroscience Methods, 134, 9-21. https://doi.org/10.1016/j.jneumeth.2003.10.009 (Original work published)

Delplanque, S., Silvert, L., Hot, P., \& Sequeira, H. (2005). Event-related P3a and P3b in response to unpredictable emotional stimuli. Biological Psychology, 68(2), 107-120. https://doi.org/10.1016/j.biopsycho.2004.04.006 (Original work published) 
542 Fox, E., Lester, V., Russo, R., Bowles, R. J., Pichler, A., \& Dutton, K. (2000). Facial

543 Expressions of Emotion: Are Angry Faces Detected More Efficiently? Cognition \&

$544 \quad$ Emotion, 14(1), 61-92. https://doi.org/10.1080/026999300378996 (Original work

$545 \quad$ published)

546 Huang, P., Xuan, X., Qiu, R., \& Liu, G. (2012). Statistical analysis on production safety 950-958. https://doi.org/10.1016/j.proeng.2012.08.264 (Original work published)

Isreal, J. B., Chesney, G. L., Wickens, C. D., \& Donchin, E. (1980). P300 and Tracking

Keil, A., Bradley, M. M., Hauk, O., Rockstroh, B., Elbert, T., \& Lang, P. J. (2002). Large-scale neural correlates of affective picture processing. Psychophysiology, 39(5), 641-649. https://doi.org/10.1111/1469-8986.3950641 (Original work published)

Lang, P. J., Bradley, M. M., \& Cuthbert, B. N. (1990). Emotion, Attention, and the Startle Reflex. Psychological Review, 97(3), 377-395. https://doi.org/10.1037/0033-295X.97.3.377 (Original work published)

Lang, P.J., Bradley, M.M., \& Cuthbert, B.N., (1997). Motivated attention: affect, activation, and action. In: Lang, P.J., Simons, R.F., Balaban, M.T. (Eds.), Attention and Orienting: Sensory and Motivational Processes. Lawrence Erlbaun Associates Publishers, Manhwah: New Jersey, pp. 97-135. 
Lang, P. J., Bradley, M. M., \& Cuthbert, B. N. (2005). International affective picture system (IAPS): Instruction manual and affective ratings. Technical Report A-6, The Center for Research in Psychophysiology, University of Florida.

Lang, P. J., Davis, M., \& Öhman, A. (2000). Fear and anxiety: Animal models and human cognitive psychophysiology. Journal of Affective Disorders, 61(3), 137-159. https://doi.org/10.1016/S0165-0327(00)00343-8 (Original work published)

Liu, P., Cao, R., Chen, X., \& Wang, Y. (2017). Response inhibition or evaluation of danger? An event-related potential study regarding the origin of the motor interference effect from dangerous objects. Brain Research, 1664, 63-73. https://doi.org/10.1016/j.brainres.2017.03.025 (Original work published)

Öhman, A., Flykt, A., \& Esteves, F. (2001). Emotion drives attention: Detecting the snake in the grass. Journal of Experimental Psychology: General, 130(3), 466-478. https://doi.org/10.1037/0096-3445.130.3.466 (Original work published)

Polich, J. (2007). Updating P300: An integrative theory of P3a and P3b. Clinical Neurophysiology, 118(10), 2128-2148. https://doi.org/10.1016/j.clinph.2007.04.019 (Original work published)

Robinson, M. D., Storbeck, J., Meier, B. P., \& Kirkeby, B. S. (2004). Watch out! That could be dangerous: Valence-arousal interactions in evaluative processing. Personality and Social Psychology Bulletin, 30(11), 1472-1484. https://doi.org/10.1177/0146167204266647 (Original work published)

Russell, J. A. (1980). A circumplex model of affect. Journal of Personality and Social 
Psychology, 39(6), 1161-1178. https://doi.org/10.1037/h0077714 (Original work published)

585

Sabatinelli, D., Lang, P. J., Keil, A., \& Bradley, M. M. (2007). Emotional perception:

Correlation of functional MRI and event-related potentials. Cerebral Cortex, 17(5), 10851091. https://doi.org/10.1093/cercor/bhl017 (Original work published)

Schupp, H. T., Junghöfer, M., Weike, A. I., \& Hamm, A. O. (2003). Attention and emotion: An ERP analysis of facilitated emotional stimulus processing. NeuroReport, 14(8), 1107-1110. https://doi.org/10.1097/00001756-200306110-00002 (Original work published)

Tipples, J., Young, A. W., Quinlan, P., Broks, P., \& Ellis, A. W. (2002). Searching for threat. Quarterly Journal of Experimental Psychology Section A: Human Experimental Psychology, 55(3), 1007-1026. https://doi.org/10.1080/02724980143000659 (Original work published)

Yuan, J., Zhang, Q., Chen, A., Li, H., Wang, Q., Zhuang, Z., \& Jia, S. (2007). Are we sensitive to valence differences in emotionally negative stimuli ? Electrophysiological evidence from an ERP study. Neuropsychologia, 45, 2764-2771. https://doi.org/10.1016/j.neuropsychologia.2007.04.018 (Original work published)

Zsido, Andras N., Matuz, A., Inhof, O., Darnai, G., Budai, T., Bandi, S., \& Csatho, A. (2019). Disentangling the facilitating and hindering effects of threat-related stimuli - A visual search study. British Journal of Psychology, 7624, 665-682. https://doi.org/10.1111/bjop.12429 (Original work published)

Zsido, Andras Norbert, Bernath, L., Labadi, B., \& Deak, A. (2020). Count on arousal: introducing a new method for investigating the effects of emotional valence and arousal on 
605 visual search performance. Psychological Research, 84(1), 1-14.

606 https://doi.org/10.1007/s00426-018-0974-y (Original work published) 


\section{Figure 1}

Topographic plots of the P3 components.

Grand-average topographic plots were calculated based on the mean amplitude in the 300to 500-ms time window for the Go trials and in the 400- to 600-ms time window for the NoGo trials as a function of valence (negative, positive and neutral) and arousal (medium and high). 


\section{Go trials NoGo trials}

negative \& low
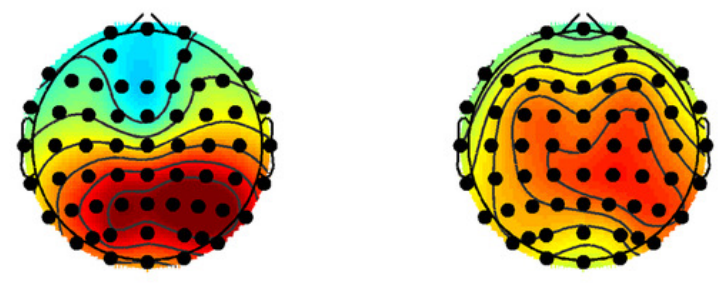

negative \& high
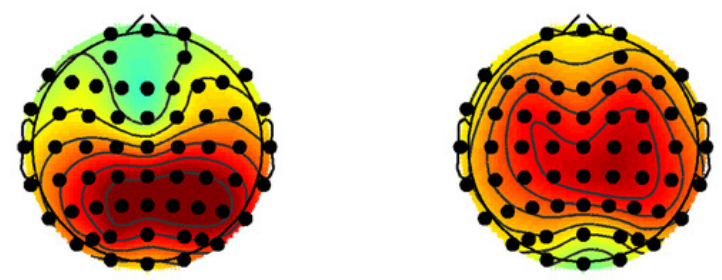

positive \& low
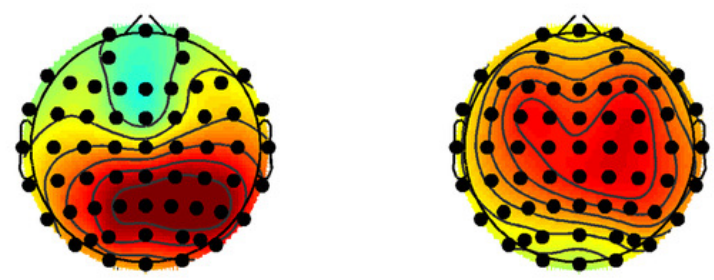

positive \& high
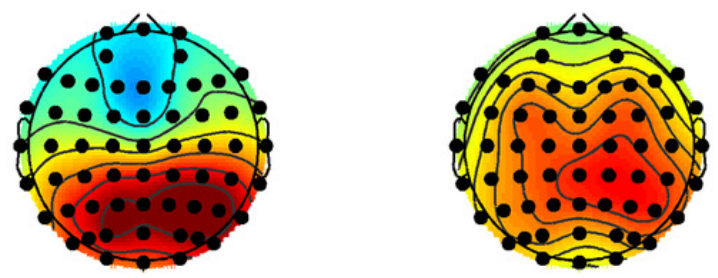

neutral \& low
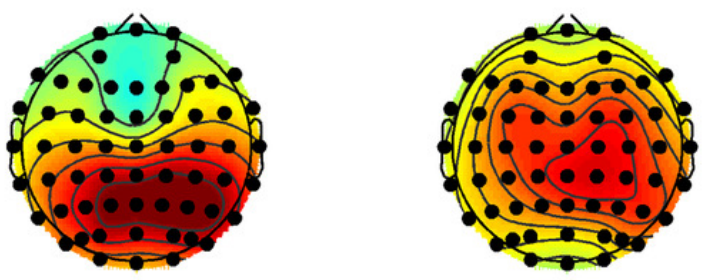

neutral \& high
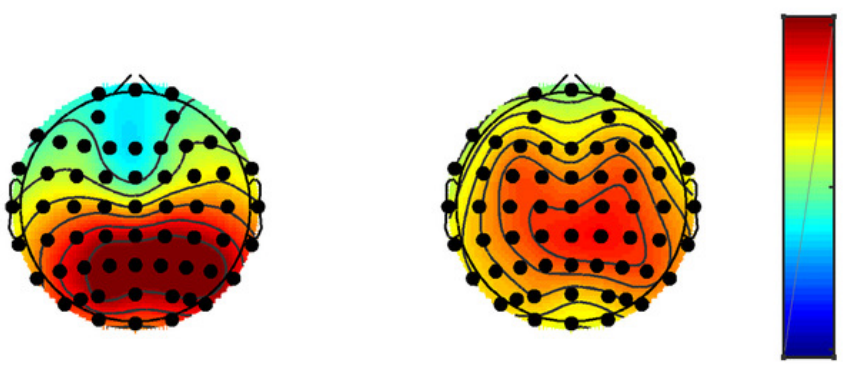
Figure 2

Results of the reaction times.

The figure presents the mean reaction times as a function of valence (negative, positive and neutral) and arousal (medium and high) in Go trials.

\section{Reaction times (ms)}

510

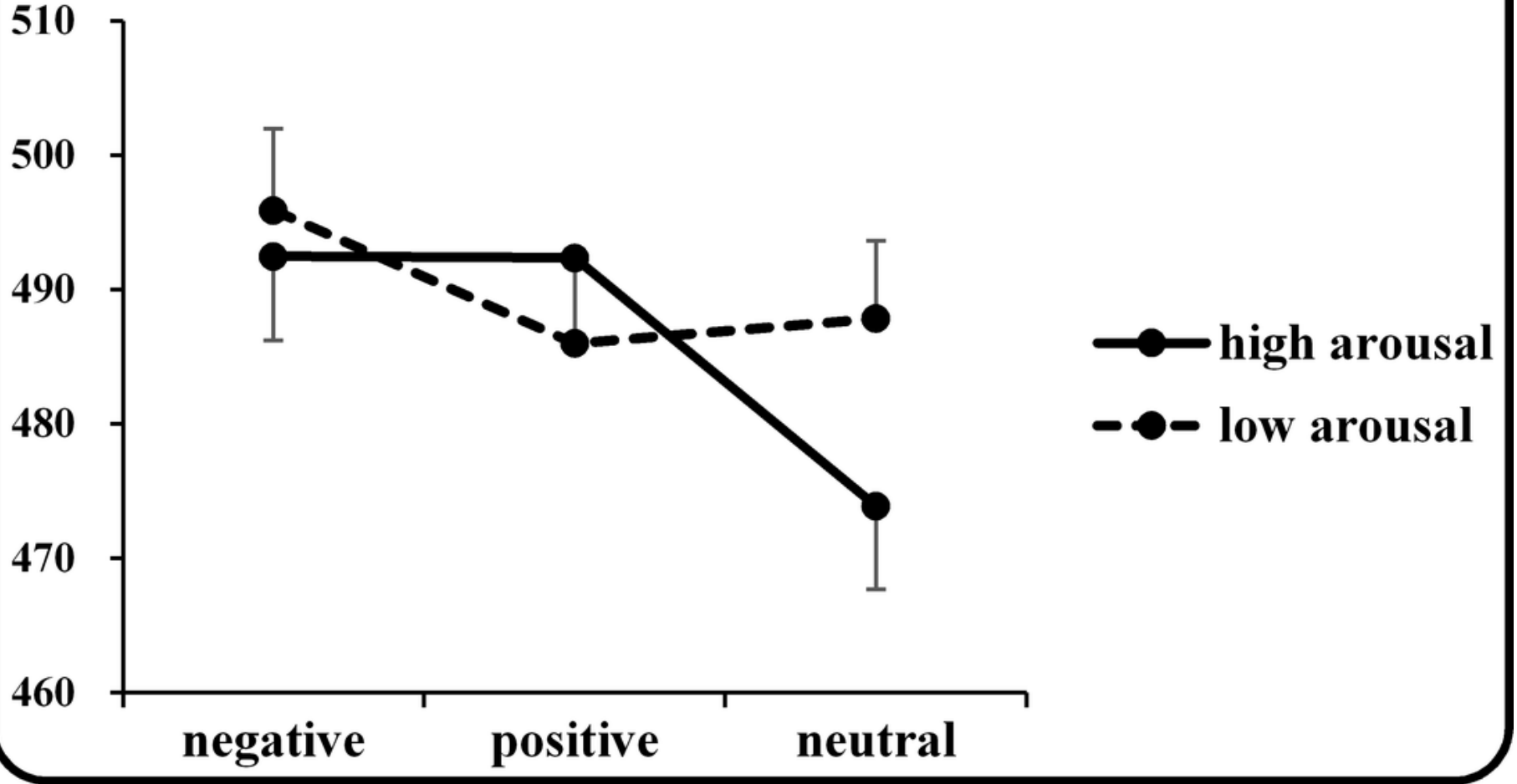


Figure 3

Results of the error rates.

The figure presents the mean error rates as a function of valence (negative, positive and neutral), arousal (medium and high) and Go/NoGo factor (Go and NoGo).

\section{Error rates (\%)}

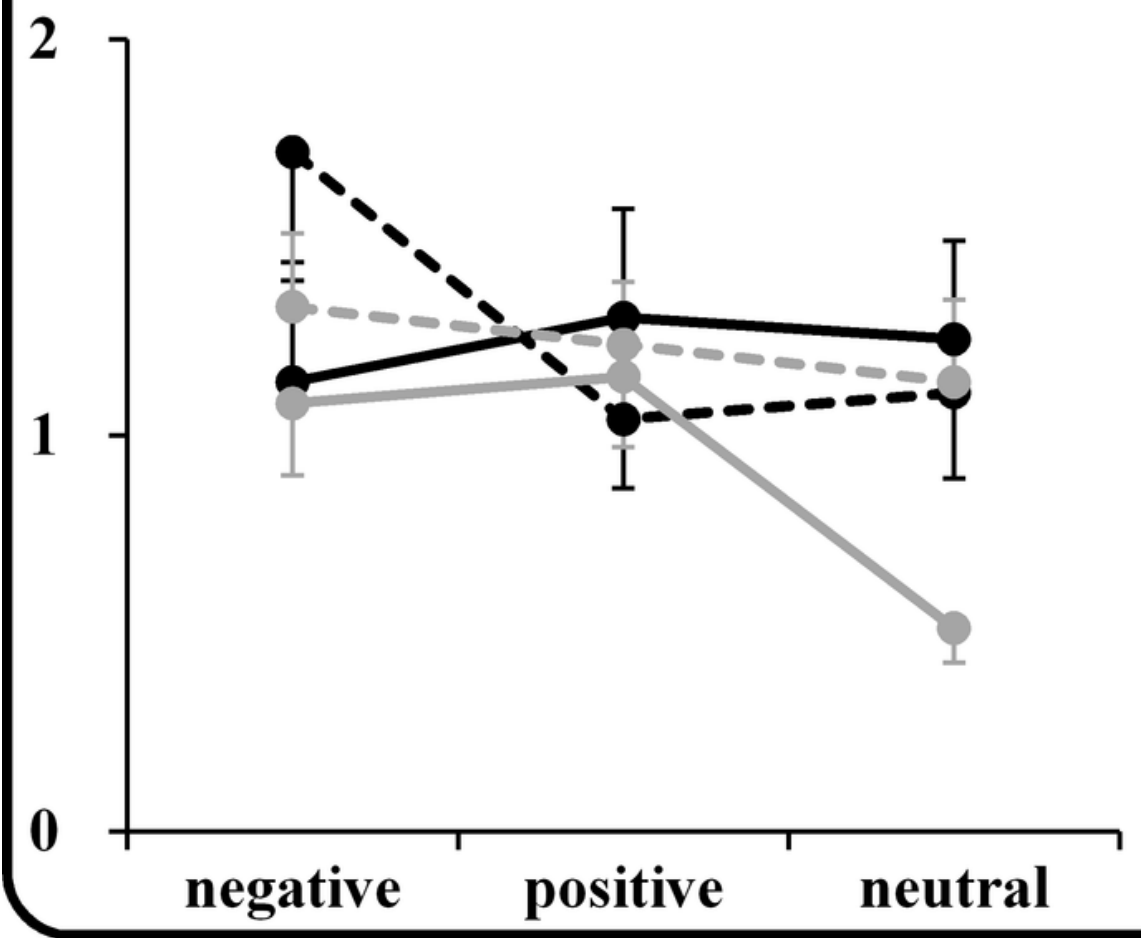

- go \& high arousal

- - - go \& low arousal nogo \& high arousal $-\infty$ nogo \& low arousal 
Figure 4

Grand-average target-locked ERPs of the P3 components.

Grand-average target-locked ERPs for Go and NoGo trials were separately presented as a function of valence (negative, positive and neutral) and arousal (medium and high) at the centroparietal area (the average of the $\mathrm{C} 5, \mathrm{C} 3, \mathrm{C} 1, \mathrm{Cz}, \mathrm{C} 2, \mathrm{C} 4, \mathrm{C} 6, \mathrm{CP} 5, \mathrm{CP} 3, \mathrm{CP} 1, \mathrm{CP}$, CP2, CP4, CP6, P5, P3, P1, Pz, P2, P4 and P6 electrodes). The rectangles filled with oblique lines indicate the analyzed time windows for the P3 amplitudes.
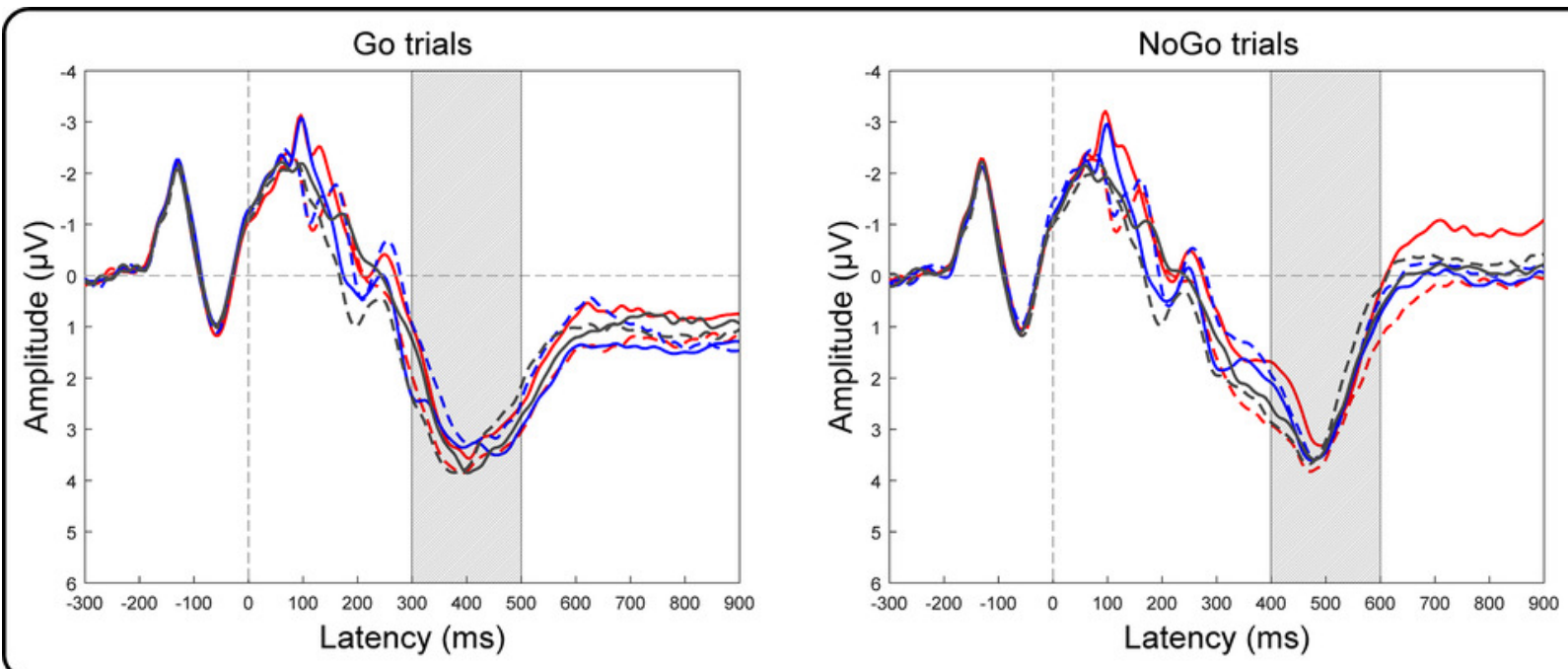

negative \& low negative \& high positive \& low positive \& high neutral \& low $-------\div$ neutral \& high 


\section{Table 1 (on next page)}

IAPS codes for target pictures in each valence and arousal level. 


\begin{tabular}{llll}
\hline & Negative valence & Neutral valence & Positive valence \\
\hline \multirow{2}{*}{ Low arousal } & 1230 (Spider) & 1121 (Lizard) & 1463 (Kittens) \\
& 1275 (Roaches) & 1947 (Octopus) & 1590 (Horse) \\
& 1019 (Snake) & 1560 (Hawk) & 1650 (Jaguar) \\
High arousal & 1205 (Spider) & 1640 (Coyote) & 1710 (Puppies) \\
& 1301 (Dog) & 1726 (Tiger) & 1720 (Lion) \\
\hline
\end{tabular}

1 


\section{Table 2 (on next page)}

Means and standard deviations of valence and arousal scores as a function of valence and arousal. The raw scores are derived from Table 1 in Lang et al. (2005). 


\begin{tabular}{|c|c|c|c|c|c|c|}
\hline & \multicolumn{2}{|c|}{ Negative valence } & \multicolumn{2}{|c|}{ Neutral valence } & \multicolumn{2}{|c|}{ Positive valence } \\
\hline & valence scores & arousal scores & valence scores & arousal scores & valence scores & arousal scores \\
\hline Low arousal & $3.69(0.40)$ & $4.81(0.04)$ & $5.76(0.10)$ & $4.52(0.27)$ & $7.26(0.17)$ & $4.69(0.13)$ \\
\hline High arousal & $3.77(0.16)$ & $5.78(0.01)$ & $5.68(0.78)$ & $5.62(0.56)$ & $7.26(0.94)$ & $5.65(0.50)$ \\
\hline
\end{tabular}

1 